\title{
Cytosolic DNA sensors regulating type I interferon induction
}

Sinead E. Keating ${ }^{1}$, Marcin Baran ${ }^{1,2}$ and Andrew G. Bowie ${ }^{1,2}$

${ }^{1}$ School of Biochemistry and Immunology and ${ }^{2}$ Immunology Research Centre, Trinity

College Dublin, Dublin 2, Ireland.

Corresponding author: Bowie, A.G. (agbowie@tcd.ie) 


\begin{abstract}
Type I interferon induction is a critical anti-pathogen response mediated by innate immune stimulation. While it has been appreciated for some time that the presence of pathogen DNA within a cell leads to a type I interferon response, it is only in the past few years that some of the key signalling proteins and DNA sensors that regulate this response have been uncovered. Here we review the nature of these DNA sensors, which includes a new family of pattern recognition receptors termed the 'AIM2-like receptors (ALRs), and consider the implications of their discovery for understanding emerging principles of innate immune DNA sensing. Furthermore, we discuss how their discovery provides a rationale as to why accumulation of self-DNA mediates IFN-dependent autoimmunity.
\end{abstract}

\title{
The type I interferon response to DNA
}

The innate immune system responds to the presence of pathogens or danger by recognizing pathogen-associated molecular patterns (PAMPs) or damage-associated molecular patterns (DAMPs) respectively, leading to altered gene expression, immune effector mechanisms and ultimately a return to homeostasis. PAMPs and DAMPs are detected by a number of classes of host pattern recognition receptors (PRRs) including the Toll-like receptors (TLRs), RIG-like receptors (RLRs), NOD-like receptors (NLRs) and C-type lectin receptors (CLRs). The induction of both proinflammatory cytokines and type I interferons (IFNs) are essential components of the PRR response. As such, chronic or inappropriate activation of PRRs is linked to many inflammatory and autoimmune diseases, which provides a strong rationale to fully elucidate PAMP and DAMP sensing mechanisms by PRRs. 
It is well known that microbial nucleic acids trigger the induction of type I IFNs such as IFN- $\alpha$ and IFN- $\beta$, and that this represents a key host defence strategy to limit the replication of invading micro-organisms. Innate immune responses to self-DNA have been linked to autoimmunity. However, in contrast to the wealth of knowledge about how pathogen RNA is detected and discriminated (from host RNA), the cellular mechanisms of innate DNA sensing are only just emerging.

The seminal discovery that mice lacking TLR9 could not elicit an immune response to CpG DNA ${ }^{1}$ marked the first identification of an innate immune receptor for the detection of foreign DNA. TLR9 is primarily expressed in plasmacytoid dendritic cells (pDCs) and B cells, and is one of a subset of nucleic acid-specific TLRs that signal from within endosomes. TLR9-independent DNA sensing mechanisms also exist. This was demonstrated with reports in mice showing that uncontrolled IFN- $\beta$ induction and ensuing lethality associated with DNAse II deficiency are not reversed in the absence of TLR9 or its downstream signalling adaptor MyD88 ${ }^{2}$. Also, DNAassociated systemic lupus erythematosus (SLE) auto-antigens trigger DC activation via TLR9-independent pathways ${ }^{3,4}$. Intracellular delivery of mammalian or bacterial dsDNA, using cationic liposomal transfection reagents, triggered macrophage and DC activation accompanied by potent TNF- $\alpha$ and IL-6 release independently of TLR ${ }^{5,6}$. This and other work hinted that innate DNA sensing was not confined to endosomes, but could also occur in the cytosol, and it is now appreciated that intracellular DNA is likely to constitute a major PAMP following infection with DNA viruses and intracellular bacteria such as Listeria monocytogenes and Legionella pneumophila ${ }^{7}$. 
In this review, we discuss recently identified cytosolic innate immune DNA sensors and how these sensors signal. Furthermore, we define a new family of PRRs involved in DNA sensing, termed the AIM2-like receptors (ALRs). The discovery of the ALRs together with other novel DNA sensors reveals that multiple DNA sensors exist, many of which operate in a cell-type specific manner, and that these DNA sensors are unlikely to discriminate between self and non-self DNA, thus providing a rationale for their likely roles in autoimmune responses to self-DNA.

\section{Signalling components involved in the cytosolic DNA response}

Although the specific receptor molecules involved in triggering TLR9-independent DNA sensing pathways were unknown until recently, studies examining the cellular responses induced by transfecting different types of DNA molecules into cells, or infecting cells with DNA viruses, uncovered a number of signalling proteins functioning downstream of DNA sensors that are required for cytosolic DNA responses (see Figure 1). Apart from the transcription factors NF- $\kappa \mathrm{B}$ and IFN regulatory factor 3 (IRF3), strong evidence exists for a central role for TANK-binding kinase-1 (TBK1) and stimulator of IFN genes (STING) in DNA-dependent pathogen responses.

\section{TBK-1 and IRF3}

In 2006, TBK1 was shown to be required for the synthetic DNA poly(dA:dT) to induce IFN- $\beta$ production via activation of IRF3 ${ }^{8}$. TBK1 directly phosphorylates and thus activates IRF3, in most cells types examined to date. Similar to poly(dA:dT), a synthetic 45 bp non-CpG DNA oligomer (termed ISD) enhanced type I IFN expression in an IRF3-dependent manner ${ }^{9}$. Type I IFN responses to the DNA viruses 
herpes simplex virus 1 (HSV-1) and murine gammaherpesvirus 68 (MHV-68), are also TBK1-dependent ${ }^{10}$, as is the immune response to DNA-based vaccines ${ }^{11}$. IRF3 also conferred resistance to MHV-68 infection in vivo ${ }^{10}$ providing evidence for a crucial role of a TBK1-IRF3 axis in the IFN response to DNA viruses. Transfected DNA triggers IRF3 and IFN- $\beta$ promoter activation via a TBK1/IKKe dependent

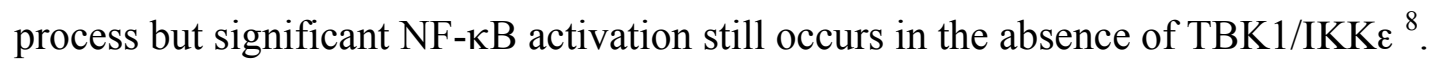

\section{STING}

STING was identified as a transmembrane-spanning endoplasmic reticulum-resident protein essential for IFN- $\beta$ induction by exogenous DNA ligands and also by $L$. monocytogenes and HSV-1 infection ${ }^{12}$. An immune function for STING was discovered independently by four groups and as such it is also known as MITA ${ }^{13}$, MPYS ${ }^{14}$ and ERIS ${ }^{15}$ (while its gene name is Tmem173). A follow-up study in STING-deficient mice showed impaired IFN induction in response to bacterial, viral and mammalian DNA, as well as to a number of DNA viruses including HSV-1, human cytomegalovirus (HCMV), vaccinia virus (VACV) and baculovirus ${ }^{16}$. Further, survival of mice lacking STING was reduced following infection with HSV1. STING functions 'upstream' of TBK1 in DNA sensing, and like TBK1, is also required for a normal immune response to DNA vaccines ${ }^{16}$. Thus, STING is critical for DNA-mediated signalling to enhance adaptive immune responses. While STING over-expression mediates potent IRF3 induction, the corresponding NF- $\mathrm{\kappa B}$ activation is not very strong ${ }^{12}$. Thus, the question of whether intracellular DNA triggers a STING-independent pathway to NF- $\mathrm{BB}$ activation warrants further study. 
The gram-negative bacterium Francisella tularensis has now also been shown to trigger IFN production in macrophages in a STING-dependent manner ${ }^{17}$ and human immunodeficiency virus (HIV-1) recognition also appears to require STING ${ }^{18}$. A forward genetic screen revealed that a mutation in Tmem173 (encoding STING) led to impaired recognition of cyclic-di-nucleotides (which are produced during $L$. monocytogenes infection), placing STING downstream not only of L. monocytogenes DNA recognition but also of L. monocytogenes cyclic-di-nucleotide ligands ${ }^{19}$. The identity of the upstream sensor for these type I IFN-inducing bacterial nucleotide ligands is currently a major focus. Finally, it has recently been shown that human SNPs in STING render cells defective in sensing DNA, emphasising the importance of STING to DNA responses in humans ${ }^{20}$.

\section{Novel intracellular DNA sensors for IFN induction}

Neither TBK1 nor STING directly recognise DNA in the cytosol. Rather, upstream cytosolic DNA sensors have recently been described, which are proposed to have the capacity to bind directly to DNA, and engage signalling pathways converging on NF$\kappa \mathrm{B}$ and IRF activation, leading to type I IFN induction (Figure 1). These sensors display some selectivity in terms of cell type, the pathogens sensed, and the exact nature of the DNA ligand tested (Table 1). However, work to date suggests that it is unlikely that these diverse sensors display differential selectivity for different types of DNA. Rather they may all simply sense cytosolic dsDNA in different cell types.

\section{DAI}

The identification of DNA-dependent activator of IFN-regulatory factors (DAI; also known as Z-DNA binding protein or ZBP-1) in 2007 represented the first 
identification of a TLR9-independent sensor for intracellular DNA ${ }^{21}$. DAI directly interacts with DNA and over-expression of DAI in murine L929 cells enhanced IFN$\beta$ induction triggered by transfected poly(dA:dT). Also, HCMV-induced IFN- $\beta$ in human fibroblasts is dependent on DAI ${ }^{22}$. The role of DAI in DNA sensing pathways, however, may be restricted to certain cell types because inhibiting DAI expression has limited effects on poly(dA:dT)-induced IFN- $\beta$ in MEFs ${ }^{23}$. Furthermore, in contrast to TBK-1 and STING-deficient mice, mice lacking DAI displayed normal immune responses to DNA virus infection and DNA vaccines ${ }^{11}$. The receptor interacting proteins, RIP1 and RIP3 facilitate NF- $\mathrm{kB}$ activation by DAI (Figure 1) ${ }^{24,25}$. Whether they regulate NF- $\kappa \mathrm{B}$ activation by other DNA sensing pathways is an open question.

\section{RNA polymerase III}

Some of the redundancy in DAI-mediated sensing of poly(dA:dT) can be attributed to the indirect sensing of this AT-rich DNA by RNA polymerase III. In 2009, RNA polymerase III was shown to transcribe AT-rich dsDNA into 5'triphosphatecontaining dsRNA which acts as a ligand for the RNA sensor retinoic acid inducible gene I (RIG-I) ${ }^{26,27}$. Since MAVS is a downstream adaptor molecule for RIG-I signalling, this explains observations that MAVS-dependent signalling is sometimes required for DNA sensing (Figure 1) ${ }^{8,28}$. The RNA polymerase III-dependent pathway has been implicated in sensing Epstein Barr virus (EBV)-encoded EBER RNA as well as HSV-1, adenovirus and L. pneumophila DNA. However, other studies have shown that RNA polymerase III is dispensible for IFN- $\beta$ induction by HSV-1 ${ }^{29,30}$ and L. pneumophila ${ }^{31}$. RNA has been proposed as the primary IFNinducing ligand in L. pneumophila infection of BMDMs and both RIG-I and Mda5 
(an RLR related to RIG-I) have been implicated in this response ${ }^{31}$. Given the importance of $\mathrm{pDCs}$ in responding to DNA, it may be that DNA contributes more significantly to the L. pneumophila-induced IFN response in these cells. RNA polymerase III appears to operate in a number of cell types, and the response to poly(dA:dT) in human cell lines is at least partially attributable to this pathway ${ }^{26,27,29}$. However, the physiological relevance of the RNA polymerase III pathway in the context of a whole animal model of pathogen infection will be difficult to assess, because RNA polymerase III is essential for normal cell function.

\section{LRRFIP1}

In 2010, using a synthetic siRNA library screen in mouse primary peritoneal macrophages, the leucine-rich repeat (LRR)-containing protein, LRRFIP1, was implicated in the induction of IFN- $\beta$ in L. monocytogenes infection, and also in response to transfected DNA ${ }^{32}$. LRRFIP1 was found to regulate a very specific $\beta$ catenin-dependent pathway whereby rather than mediating NF-אB or IRF3 activation, LRRFIP1 triggered phosphorylation and subsequent nuclear translocation of $\beta$-catenin, which then interacted with IRF3 (Figure 1). This facilitated p300 recruitment and histone acetylation at the ifnb1 promoter locus. LRRFIP1 was proposed to directly recognise DNA because it immunoprecipitated with synthetic dsDNA. However, physiologically relevant direct DNA binding remains to be demonstrated. Furthermore, LRRFIP1 regulated a highly specific pathway to IFN- $\beta$ induction utilising $\beta$-catenin in response to both DNA and RNA ligands while having no effect on L. monocytogenes- or DNA-mediated TNF- $\alpha$ induction. 
Very recently, in pDCs a TLR9-independent, but MyD88-dependent CpG DNA sensing mechanism led to the discovery of a role for the DEAD/H-box helicases, DHX9 and DHX36, in CpG-dependent gene induction ${ }^{33}$. DHX9 and DHX36 were initially identified as CpG-interacting proteins by mass spectrometry. siRNA knockdown experiments revealed that DHX36 was specifically required for the IFN- $\alpha$ response to HSV-1 infection in pDCs while DHX9 expression was primarily required for HSV-1-mediated TNF- $\alpha$ induction. Both DHX36 and DHX9 interact with the TIR domain of MyD88. Thus, it was proposed that two TLR9-independent, MyD88dependent DNA sensing pathways exist in pDCs whereby DHX36 governs a pathway specific for IRF7 activation and IFN- $\alpha$ induction while DHX9 triggers nuclear

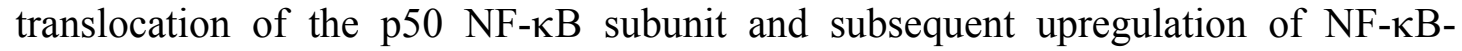
dependent genes such as TNF- $\alpha$ and IL-6.

$\mathrm{Ku} 70$

Another protein very recently implicated in DNA sensing in a specific context is Ku70. HEK293 cells are refractory to DNA-induced IFN- $\beta$ induction, except for RNA polymerase III sensing of AT-rich DNA ${ }^{26,27,29}$. However, plasmid DNA induces the type III interferon, IFN- $\lambda 1$, as opposed to type I IFN- $\alpha$ or IFN- $\beta$ in HEK293 cells ${ }^{34}$. Ku70, a protein involved in DNA repair, was identified as a dsDNA-binding protein by mass spectrometry, and using siRNA and cells from $\mathrm{Ku} 70^{-/-}$mice subsequently implicated as the sensor responsible for triggering this pathway. DNAinduced IFN- $\lambda 1$ induction correlated with binding of IRF1 and IRF7, but not NF- $\mathrm{KB}$ or IRF3, to the IL29 (IFN- $\lambda 1$ ) promoter, suggesting that Ku70 might engage a signalling pathway regulating IRF1 and IRF7 activation. As Ku70-mediated IFN- $\lambda 1$ induction required DNA of greater than $500 \mathrm{bp}$, the authors suggest that $\mathrm{Ku} 70$ 
proteins may need to bind to multiple sites along a dsDNA molecule in order to stimulate signal transduction to IRFs ${ }^{34}$.

\section{IFI16 and $p 204$}

An IFN- $\beta$-inducing 70bp dsDNA from VACV was used to affinity purify DNAbinding proteins from cytosolic extracts of human monocytes, leading to the identification of IFI16 ${ }^{29}$. IFI16 was found to recruit STING to activate a TBK1-IRF3dependent pathway to IFN- $\beta$ induction, while siRNA showed that both DNA and HSV-1-induced IFN- $\beta$ was IFI16-dependent. Interestingly, IFI16 is part of a larger protein family termed the pyrin and HIN domain (PYHIN) family, of which there are four human and 13 murine members (Figure 2). The closest murine structural counterpart to IFI16, p204, is the only murine PYHIN family member that, like IFI16, contains two HIN200 DNA binding domains and a pyrin domain (Figure 2). Inhibition of p204 expression in murine cells showed that p204 was essential for IRF3 and NF- $\kappa \mathrm{B}$ activation, and type I IFN and pro-inflammatory gene induction driven by HSV-1 infection or by dsDNA oligonucleotides. A prerequisite for a bona fide DNA sensor is a demonstration of direct binding to immune-stimulatory DNA and as such, IFI16 not only colocalised with DNA in intact cells, but purified IFI16 protein also directly bound DNA in vitro ${ }^{29}$.

Another member of the PYHIN family, AIM2, is a cytosolic DNA receptor that forms an inflammasome with ASC to trigger caspase 1 activation ${ }^{17,35-37}$. Very recently, IFI16 has also been proposed to mediate inflammasome activation, in response to Kaposi's sarcoma-associated virus (KSHV) infection of endothelial cells ${ }^{38}$. In this case IFI16 was proposed to sense viral DNA in the nucleus, raising the possibility that 
IFI16 may have alternative DNA sensing functions in the nucleus and the cytosol. Recent studies with AIM2, IFI16 and p204 suggest the existence of a brand new family of PRRs, previously unappreciated, and termed 'AIM2-like receptors' (ALRs). Table 2 compares the characteristics of the DNA sensing ALRs with the RNA sensing RLRs. It will be of great interest to assess the role of the other PYHIN family members (Figure 2) in innate immune responses to DNA, which may be easier to address in the human system, given that two out of the four known human PYHIN proteins have already been assigned innate DNA sensing roles. As PYHIN proteins have other defined roles in cellular processes ${ }^{39-41}$, they may be analogous to the NOD proteins whereby some family members function as PRRs (the NLRs) and some do $\operatorname{not}^{42}$.

\section{Negative regulation of intracellular DNA sensing pathways}

The inappropriate induction of type I IFNs leads to the on-set of such auto-immune disorders as SLE and Aicardi-Goutieres syndrome (AGS). The detrimental effects of dysregulated type I IFN production is underscored by the fatal encephalopathy induced by overproduction of IFN- $\alpha$ in AGS ${ }^{43}$. Further, irf3 or ifn $\alpha \beta R$ deletion can

reverse the lethal phenotype of autoimmune disorders in mice ${ }^{44-46}$. Accordingly, inappropriately generated self nucleic acid must be cleared efficiently in order to prevent autoimmune responses. The existence of the cytosolic DNA sensing pathways mediating IFN induction described above provide a rationale for this, since they do not seem to recognise any particular structural motif of DNA that would discriminate between self and non-self. It may be that cytosolic DNA sensing pathways evolved to sense 'danger' (such as free dsDNA in the cytosol) more so than 'stranger' (the presence of a pathogen), whereas cytosolic RNA sensing by the RLRs does seem 
capable of detecting structural moieties specific to pathogen RNA, such as 5'triphosphates (Table 2).

A role for host DNases in the elimination of mislocalised host DNA to prevent autoimmunity is emerging. Mice deficient in DNase I, the most abundant nuclease found in serum, develop SLE-like autoimmune disorders ${ }^{38}$ while mutation of the DNase I gene has been associated with SLE in humans ${ }^{47,48}$. DNase II plays a major role in the clearance of DNA from apoptotic cells within macrophage phagosomes. In mice lacking DNase II, accumulating DNA from phagocytosed apoptotic cells drives excessive IFN- $\beta$ and TNF production inducing embryonic lethality ${ }^{49}$. Further, in DNase II-deficient mice, DNA released from erythroid precursor cells during erythrocyte development is not properly digested and so accumulates in macrophage lysosomes within foci of erythropoiesis known as blood islands ${ }^{50}$. This triggers constitutive IFN- $\beta$ production in the liver along with an ensuing upregulation of IFNinducible genes. These embryos suffer severe anaemia and die in utero. However, DNase II-IFN- $\alpha / \beta$ R double deficient mice developed normally and appeared healthy despite the abnormal accumulation of DNA in blood-island macrophages confirming the contribution of uncontrolled DNA-induced IFN to the lethality observed.

Mutations in human cytosolic $3^{\prime}-5^{\prime}$ exonuclease Trex1 (DNase III) are associated with both SLE and AGS. Trex1 deficiency in mice manifests in the development of lethal autoimmunity due to excessive type I IFN and auto-antibody production ${ }^{44}$. Cytosolic ssDNA derived from endogenous retroelements were shown to accumulate in cells lacking Trex1 leading to aberrant type I IFN induction. Whether a specific cytosolic sensor that triggers type I IFN induction in response to these ssDNA Trex1 
substrates exists remains to be elucidated. Such a ssDNA sensor may be central to HIV-1 innate immunity as HIV was found to subvert Trex 1 activity to rapidly degrade ssDNA intermediates generated during reverse transcription of this retroviral genome 18. Given the link between nuclease deficiency and autoimmunity, it is of interest to determine which, if any, of the new DNA sensors described above are responsible for responding to endogenous DNA in contexts where nucleases are deficient.

Due to the expression of high basal levels of IRF7, pDCs are the most potent IFN$\alpha$-producing cells (reviewed in ${ }^{51}$ ). The production of type I IFN by pDCs is a key component of the pathogenesis of many autoimmune diseases. Polymorphisms in genes of the type I IFN signalling system, including IRF5 and STAT4, have been identified as susceptibility factors in many autoimmune diseases, including Sjogren's syndrome (SS) and SLE ${ }^{52-55}$. Serum levels of type I IFN are increased ${ }^{56}$ while blood pDC populations are reduced and infiltrating pDCs are enhanced in the salivary glands in SS patients ${ }^{57}$. Similarly, in SLE patients, circulating pDCs are significantly lower ${ }^{58}$ due to migration of these cells to skin ${ }^{59,60}$ and renal tissue ${ }^{61}$. Nucleoproteins and immunoglobulins complexed to self DNA have been shown to continually activate pDCs to produce type I IFN in SLE ${ }^{62,63}$. This leads to myeloid DC differentiation and continual activation of autoreactive $\mathrm{T}$ cells ${ }^{64}$. Thus, any intracellular DNA sensor implicated in sensing host DNA to trigger an autoimmune response will likely be active in pDCs. Recently, dsDNA-activated human DCs were shown to be more potent than LPS- or inflammatory cytokine-activated DCs at triggering an adaptive immune response and IFI16 was found to contribute to this response ${ }^{65}$. 


\section{Viral evasion of innate DNA sensing mechanisms}

Most PRRs identified to date are subject to targeting by viral evasion mechanisms ${ }^{66}$, and this is also becoming apparent for some of the novel cytosolic DNA sensors (Table 2). Prior to the discovery of DNA sensors, it was already appreciated that multiple viral mechanisms to antagonize TBK1 signalling existed (reviewed in ${ }^{66}$ ), however it has also now been shown that some viruses also target the novel DNA sensors more directly. For example, murine CMV encodes a RHIM-containing protein, M45, which blocks DAI signalling to NF- $\mathrm{kB}{ }^{25}$. The HCMV tegument protein, pUL83, can associate with the human PYHIN proteins IFI16 and IFIX (Figure 2), and a mutant virus with impaired pUL83 expression was more susceptible to type I IFN treatment ${ }^{67}$. Thus, IFI16 and/or IFIX targeting by HCMV may represent a strategy to evade innate sensing of its dsDNA genome. The poxvirus-encoded M13L protein contains an N-terminal pyrin domain and interacts with ASC to antagonise caspase 1 activation and subsequent IL1 $\beta$ processing ${ }^{68}$. Disruption of the M13L gene in myxoma virus gave rise to an attenuated virus displaying reduced disease progression in rabbits due to a potent inflammatory response to infection. Thus, M13L-expressing poxviruses can potentially antagonise inflammasome activation triggered by AIM2 upon sensing viral DNA. If this viral immunomodulatory protein targets the pyrin domain of the other PYHIN family members, for example IFI16, this may represent a very clever viral strategy evolved by poxviruses to simultaneously suppress two arms of the innate machinery for sensing viral DNA.

\section{DNA sensing: future perspectives and outstanding questions}


Understanding of innate intracellular DNA sensing pathways has advanced rapidly in recent years. As described above, a number of candidate sensors of intracellular DNA have now been described. However, key questions remain to be addressed. Whether all of the current described DNA sensors are actually true receptors requires clarification. For example, specific regulation of IFN- $\beta$ by LRRFIP1 independently of any role in TNF- $\alpha$ induction coupled with no role in IRF3 or p65 activation points to a more downstream role for LRRFIP1 in nucleic acid mediated IFN- $\beta$ induction. Consistent with this, infection with both VSV (an RNA virus) and L. monocytogenes, as well as synthetic dsRNA and dsDNA analogues appear to activate this pathway ${ }^{32}$, suggesting LRRFIP1 may regulate multiple, and not just DNA sensing, PRR pathways to type I IFN transcriptional activation. Furthermore, DHX9 is required for DNA-mediated NF- $\kappa$ B activation only, while DHX36 mediates IRF7 activation in response to DNA but is not required for NF-אB induction ${ }^{33}$. How these molecules act as separate sensors upstream of MyD88 to differentially activate specific transcription factors requires further examination.

The apparent redundancy of some of the DNA sensors raises the question of which ones are physiologically relevant in sensing viral and bacterial infection in primary cells? Are specific sets of DNA sensors engaged by particular pathogens? Cell-type specificity may explain the existence of so many different pathways (Table 1).

A major question still outstanding is whether these newly described DNA sensing mechanisms function in the nucleus or whether they only detect foreign DNA in the cytosol? It would seem more rational to restrict immune surveillance for DNA to the cytosol, where its presence could alert the cell to 'danger' either in the form of a 
pathogen DNA, or mis-localised or accumulating host DNA. However, the proposal that IFI16 can sense viral DNA in the nucleus ${ }^{38}$, tied in with the fact that it, and other DNA sensors such as DHX9 and RNA polymerase III are expressed abundantly in the nucleus, may suggest that the nucleus does not enjoy immune privilege when it comes to DNA. If so, then the issue of what prevents the aberrant activation of a deleterious IFN- $\beta$ response to host chromosomal DNA will need to be resolved. As DNA sensors such as IFI16 and $\mathrm{Ku} 70$ are also implicated in DNA damage responses, they may simply signal IFN- $\beta$ induction upon encountering any type of naked DNA not complexed with protein in chromatin.

These questions pose some major challenges and addressing them will advance our understanding of how the innate immune system detects and responds not only to invading pathogens but also to self nucleic acid leading to inflammation and autoimmunity.

\section{Acknowledgements}

The authors are supported by Science Foundation Ireland.

\section{References}

1 Hemmi, H. et al. (2000) A Toll-like receptor recognizes bacterial DNA. Nature 408 (6813), 740-745

2 Okabe, Y. et al. (2005) Toll-like receptor-independent gene induction program activated by mammalian DNA escaped from apoptotic DNA degradation. $J$ Exp Med 202 (10), 1333-1339

3 Boule, M.W. et al. (2004) Toll-like receptor 9-dependent and -independent dendritic cell activation by chromatin-immunoglobulin $G$ complexes. $J$ Exp Med 199 (12), 1631-1640

4 Decker, P. et al. (2005) Nucleosome, the main autoantigen in systemic lupus erythematosus, induces direct dendritic cell activation via a MyD88-independent pathway: consequences on inflammation. $J$ Immunol 174 (6), 3326-3334 
5 Yasuda, K. et al. (2005) Macrophage activation by a DNA/cationic liposome complex requires endosomal acidification and TLR9-dependent and -independent pathways. $J$ Leukoc Biol 77 (1), 71-79

6 Yasuda, K. et al. (2005) Endosomal translocation of vertebrate DNA activates dendritic cells via TLR9-dependent and -independent pathways. J Immunol 174 (10), 6129-6136

7 Monroe, K.M. et al. (2010) Induction of type I interferons by bacteria. Cell Microbiol 12 (7), 881-890

8 Ishii, K.J. et al. (2006) A Toll-like receptor-independent antiviral response induced by double-stranded B-form DNA. Nat Immunol 7 (1), 40-48

9 Stetson, D.B. and Medzhitov, R. (2006) Recognition of cytosolic DNA activates an IRF3-dependent innate immune response. Immunity 24 (1), 93-103

10 Miyahira, A.K. et al. (2009) TANK-binding kinase-1 plays an important role during in vitro and in vivo type I IFN responses to DNA virus infections. J Immunol 182 (4), 2248-2257

11 Ishii, K.J. et al. (2008) TANK-binding kinase-1 delineates innate and adaptive immune responses to DNA vaccines. Nature 451 (7179), 725-729

12 Ishikawa, H. and Barber, G.N. (2008) STING is an endoplasmic reticulum adaptor that facilitates innate immune signalling. Nature 455 (7213), 674-678

13 Zhong, B. et al. (2008) The adaptor protein MITA links virus-sensing receptors to IRF3 transcription factor activation. Immunity 29 (4), 538550

14 Jin, L. et al. (2008) MPYS, a novel membrane tetraspanner, is associated with major histocompatibility complex class II and mediates transduction of apoptotic signals. Mol Cell Biol 28 (16), 5014-5026

15 Sun, W. et al. (2009) ERIS, an endoplasmic reticulum IFN stimulator, activates innate immune signaling through dimerization. Proc Natl Acad Sci $U S A 106$ (21), 8653-8658

16 Ishikawa, H. et al. (2009) STING regulates intracellular DNA-mediated, type I interferon-dependent innate immunity. Nature 461 (7265), 788-792

17 Jones, J.W. et al. (2010) Absent in melanoma 2 is required for innate immune recognition of Francisella tularensis. Proc Natl Acad Sci $U S A$ 107 (21), 9771-9776

18 Yan, N. et al. (2010) The cytosolic exonuclease TREX1 inhibits the innate immune response to human immunodeficiency virus type 1. Nat Immunol 11 (11), 1005-1013

19 Sauer, J.D. et al. (2011) The N-ethyl-N-nitrosourea-induced Goldenticket mouse mutant reveals an essential function of Sting in the in vivo interferon response to Listeria monocytogenes and cyclic dinucleotides. Infect Immun 79 (2), 688-694

20 Jin, L. et al. (2011) Identification and characterization of a loss-offunction human MPYS variant. Genes Immun 12 (4), 263-269

21 Takaoka, A. et al. (2007) DAI (DLM-1/ZBP1) is a cytosolic DNA sensor and an activator of innate immune response. Nature 448 (7152), 501-505

22 DeFilippis, V.R. et al. (2010) Human cytomegalovirus induces the interferon response via the DNA sensor ZBP1. $J$ Virol 84 (1), 585-598 
23 Wang, Z. et al. (2008) Regulation of innate immune responses by DAI (DLM-1/ZBP1) and other DNA-sensing molecules. Proc Natl Acad Sci U S A 105 (14), 5477-5482

24 Kaiser, W.J. et al. (2008) Receptor-interacting protein homotypic interaction motif-dependent control of NF-kappa B activation via the DNA-dependent activator of IFN regulatory factors. $J$ Immunol 181 (9), 6427-6434

25 Rebsamen, M. et al. (2009) DAI/ZBP1 recruits RIP1 and RIP3 through RIP homotypic interaction motifs to activate NF-kappaB. EMBO Rep 10 (8), 916-922

26 Ablasser, A. et al. (2009) RIG-I-dependent sensing of poly(dA:dT) through the induction of an RNA polymerase III-transcribed RNA intermediate. Nat Immunol 10 (10), 1065-1072

27 Chiu, Y.H. et al. (2009) RNA polymerase III detects cytosolic DNA and induces type I interferons through the RIG-I pathway. Cell 138 (3), 576591

28 Cheng, G. et al. (2007) Double-stranded DNA and double-stranded RNA induce a common antiviral signaling pathway in human cells. Proc Natl Acad Sci U S A 104 (21), 9035-9040

29 Unterholzner, L. et al. (2010) IFI16 is an innate immune sensor for intracellular DNA. Nat Immunol 11 (11), 997-1004

30 Melchjorsen, J. et al. (2010) Early innate recognition of herpes simplex virus in human primary macrophages is mediated via the MDA5/MAVSdependent and MDA5/MAVS/RNA polymerase III-independent pathways. $J$ Virol 84 (21), 11350-11358

31 Monroe, K.M. et al. (2009) Identification of host cytosolic sensors and bacterial factors regulating the type $I$ interferon response to Legionella pneumophila. PLoS Pathog 5 (11), e1000665

32 Yang, P. et al. (2010) The cytosolic nucleic acid sensor LRRFIP1 mediates the production of type I interferon via a beta-catenin-dependent pathway. Nat Immunol 11 (6), 487-494

33 Kim, T. et al. (2010) Aspartate-glutamate-alanine-histidine box motif (DEAH)/RNA helicase A helicases sense microbial DNA in human plasmacytoid dendritic cells. Proc Natl Acad Sci U S A 107 (34), 1518115186

34 Zhang, X. et al. (2011) Cutting edge: Ku70 is a novel cytosolic DNA sensor that induces type III rather than type I IFN. J Immunol 186 (8), 45414545

35 Hornung, V. et al. (2009) AIM2 recognizes cytosolic dsDNA and forms a caspase-1-activating inflammasome with ASC. Nature 458 (7237), 514-518

36 Fernandes-Alnemri, T. et al. (2010) The AIM2 inflammasome is critical for innate immunity to Francisella tularensis. Nat Immunol 11 (5), 385393

37 Rathinam, V.A. et al. (2010) The AIM2 inflammasome is essential for host defense against cytosolic bacteria and DNA viruses. Nat Immunol 11 (5), 395-402

38 Kerur, N. et al. (2011) IFI16 Acts as a Nuclear Pathogen Sensor to Induce the Inflammasome in Response to Kaposi Sarcoma-Associated Herpesvirus Infection. Cell Host Microbe 9 (5), 363-375 
39 Johnstone, R.W. and Trapani, J.A. (1999) Transcription and growth regulatory functions of the $\mathrm{HIN}-200$ family of proteins. Mol Cell Biol 19 (9), 5833-5838

40 Asefa, B. et al. (2004) The interferon-inducible p200 family of proteins: a perspective on their roles in cell cycle regulation and differentiation. Blood Cells Mol Dis 32 (1), 155-167

41 Duan, X. et al. (2011) Differential Roles for the Interferon-Inducible IFI16 and AIM2 Innate Immune Sensors for Cytosolic DNA in Cellular Senescence of Human Fibroblasts. Mol Cancer Res 9 (5), 589-602

42 Inohara, N. and Nunez, G. (2003) NODs: intracellular proteins involved in inflammation and apoptosis. Nat Rev Immunol 3 (5), 371-382

43 Crow, Y.J. and Rehwinkel, J. (2009) Aicardi-Goutieres syndrome and related phenotypes: linking nucleic acid metabolism with autoimmunity. Hum Mol Genet 18 (R2), R130-136

44 Stetson, D.B. et al. (2008) Trex1 prevents cell-intrinsic initiation of autoimmunity. Cell $134(4), 587-598$

45 Braun, D. et al. (2003) Type I Interferon controls the onset and severity of autoimmune manifestations in Ipr mice. J Autoimmun 20 (1), 15-25

46 Santiago-Raber, M.L. et al. (2003) Type-I interferon receptor deficiency reduces lupus-like disease in NZB mice. $J$ Exp Med 197 (6), 777-788

47 Napirei, M. et al. (2000) Features of systemic lupus erythematosus in Dnase1-deficient mice. Nat Genet 25 (2), 177-181

48 Yasutomo, K. et al. (2001) Mutation of DNASE1 in people with systemic lupus erythematosus. Nat Genet 28 (4), 313-314

49 Kawane, K. et al. (2003) Impaired thymic development in mouse embryos deficient in apoptotic DNA degradation. Nat Immunol 4 (2), 138-144

50 Yoshida, H. et al. (2005) Lethal anemia caused by interferon-beta produced in mouse embryos carrying undigested DNA. Nat Immunol 6 (1), 49-56

51 Gilliet, M. et al. (2008) Plasmacytoid dendritic cells: sensing nucleic acids in viral infection and autoimmune diseases. Nat Rev Immunol 8 (8), 594606

52 Gestermann, N. et al. (2010) STAT4 is a confirmed genetic risk factor for Sjogren's syndrome and could be involved in type 1 interferon pathway signaling. Genes Immun 11 (5), 432-438

53 Miceli-Richard, C. et al. (2007) Association of an IRF5 gene functional polymorphism with Sjogren's syndrome. Arthritis Rheum 56 (12), 39893994

54 Nordmark, G. et al. (2009) Additive effects of the major risk alleles of IRF5 and STAT4 in primary Sjogren's syndrome. Genes Immun 10 (1), 68-76

55 Sigurdsson, S. et al. (2008) Comprehensive evaluation of the genetic variants of interferon regulatory factor 5 (IRF5) reveals a novel 5 bp length polymorphism as strong risk factor for systemic lupus erythematosus. Hum Mol Genet 17 (6), 872-881

56 Mavragani, C.P. et al. (2007) Augmented interferon-alpha pathway activation in patients with Sjogren's syndrome treated with etanercept. Arthritis Rheum 56 (12), 3995-4004 
57 Gottenberg, J.E. et al. (2006) Activation of IFN pathways and plasmacytoid dendritic cell recruitment in target organs of primary Sjogren's syndrome. Proc Natl Acad Sci U S A 103 (8), 2770-2775

58 Robak, E. et al. (2006) Peripheral blood lymphocyte apoptosis and circulating dendritic cells in patients with systemic lupus erythematosus: correlation with immunological status and disease-related symptoms. Clin Rheumatol 25 (2), 225-233

59 Blomberg, S. et al. (2001) Presence of cutaneous interferon-alpha producing cells in patients with systemic lupus erythematosus. Lupus 10 (7), 484-490

60 Farkas, L. et al. (2001) Plasmacytoid dendritic cells (natural interferonalpha/beta-producing cells) accumulate in cutaneous lupus erythematosus lesions. Am J Pathol 159 (1), 237-243

61 Tucci, M. et al. (2008) Glomerular accumulation of plasmacytoid dendritic cells in active lupus nephritis: role of interleukin-18. Arthritis Rheum 58 (1), 251-262

62 Means, T.K. et al. (2005) Human lupus autoantibody-DNA complexes activate DCs through cooperation of CD32 and TLR9. J Clin Invest 115 (2), 407-417

63 Ronnblom, L. and Pascual, V. (2008) The innate immune system in SLE: type I interferons and dendritic cells. Lupus 17 (5), 394-399

64 Blanco, P. et al. (2001) Induction of dendritic cell differentiation by IFNalpha in systemic lupus erythematosus. Science 294 (5546), 1540-1543

65 Kis-Toth, K. et al. (2011) Cytosolic DNA-Activated Human Dendritic Cells Are Potent Activators of the Adaptive Immune Response. $J$ Immunol 187 (3), 1222-1234

66 Bowie, A.G. and Unterholzner, L. (2008) Viral evasion and subversion of pattern-recognition receptor signalling. Nat Rev Immunol 8 (12), $911-922$

67 Cristea, I.M. et al. (2010) Human cytomegalovirus pUL83 stimulates activity of the viral immediate-early promoter through its interaction with the cellular IFI16 protein. $J$ Virol 84 (15), 7803-7814

68 Johnston, J.B. et al. (2005) A poxvirus-encoded pyrin domain protein interacts with ASC-1 to inhibit host inflammatory and apoptotic responses to infection. Immunity $23(6), 587-598$

69 Ludlow, L.E. et al. (2005) The HIN-200 family: more than interferoninducible genes? Exp Cell Res 308 (1), 1-17

70 Parker, D. et al. (2011) Streptococcus pneumoniae DNA Initiates Type I Interferon Signaling in the Respiratory Tract. MBio 2 (3) 


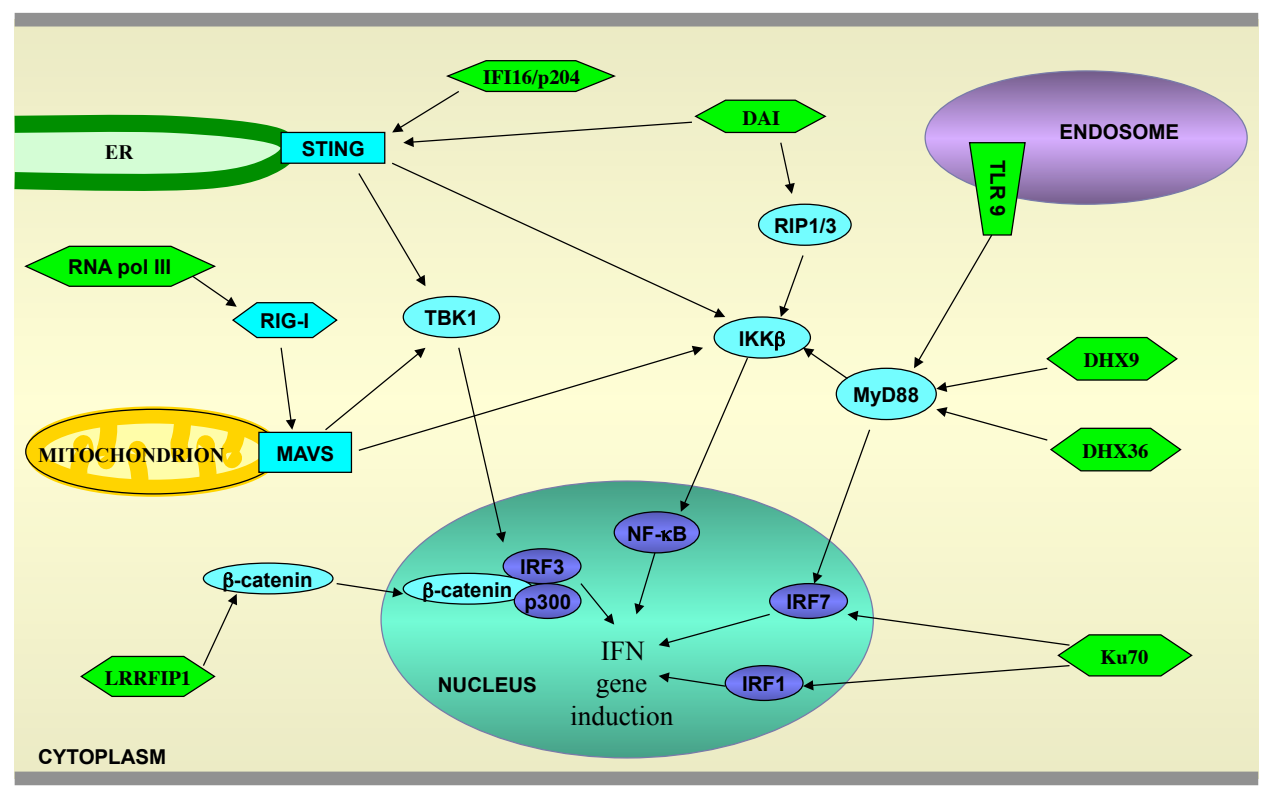

Figure 1. Cytosolic sensors of DNA mediating interferon induction.

Intracellular dsDNA produced during infection with pathogens such as dsDNA viruses and bacteria or from phagocytosed dead cells is a potent inducer of immune responses resulting in the production of proinflammatory cytokines and IFNs. A variety of intracellular sensors recognizing dsDNA (green) have been described. TLR9 recognizes CpG DNA in endosomes and triggers MyD88-dependent activation of IRF7 and NF- $\kappa B$ via IKK $\beta$. Binding of dsDNA by DAI results in STING- and TBK1-dependent IRF3 activation and induction of type I IFNs. It also activates NF$\kappa \mathrm{B}$ via RIP1 and RIP3. Human IFI16 and murine p204 induce STING-dependent activation of IRF3 and NF- $\mathrm{KB}$ transcription factors. RNA polymerase III converts AT-rich DNA into an endogenous 5' triphosphate-containing RNA ligand, which then activates RIG-I to trigger MAVS- and TBK1-dependent activation of transcription 
factors. Ku70 activates IRF1 and IRF7 to induce IFN $\lambda 1$. Cytosolic recognition of CpG-A DNA by DHX36 induces MyD88-dependent IRF7 activation and induction of IFN- $\alpha$, whereas DHX9 binds CpG-B DNA triggering MyD88-dependent activation of NF- $\kappa \mathrm{B}$ and production of proinflammatory cytokines. LRRFIP1 activation by DNA stimulates $\beta$-catenin phosphorylation at Ser552 promoting a relocalisation of $\beta$ catenin to the nucleus where it interacts with IRF3. This enhances the recruitment of the histone acetyltransferase, $\mathrm{p} 300$, to the IFN- $\beta$ enhanceosome resulting in transcriptional activation. Induction of IFNs in response to intracellular dsDNA is a key step in early immune responses to various pathogens and danger signals, and is also involved in shaping the later adaptive immune response. 
A. Human PYHIN family

\begin{tabular}{|c|c|c|}
\hline Ifi 16 & HIN A & HIN B \\
\hline MNDA & HIN A & \\
\hline IFIX/Pyhin 1 & HIN A & \\
\hline AIM2 & HIN C & \\
\hline
\end{tabular}

B. Murine PYHIN family

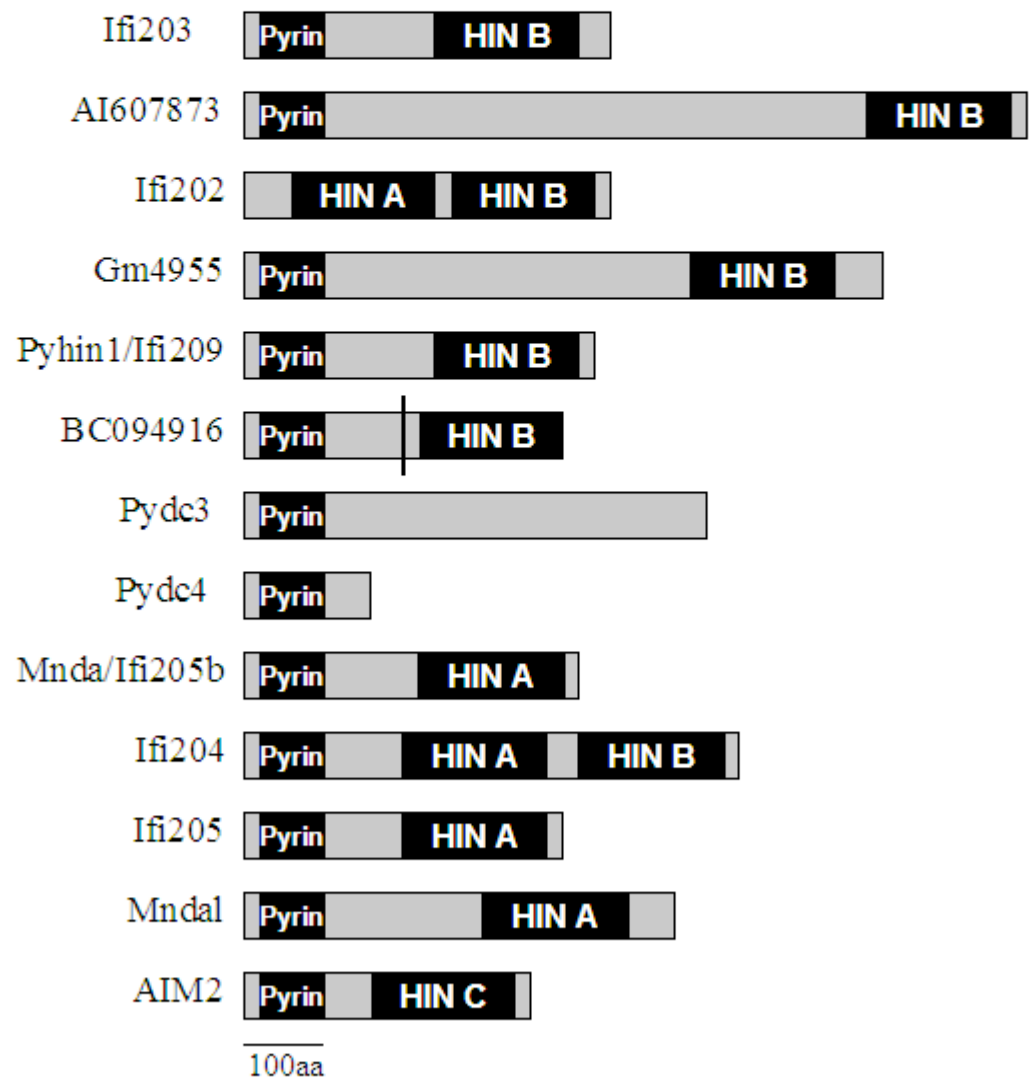

Figure 2. The human and murine PYHIN protein families.

PYHIN proteins consist of a pyrin domain and at least one HIN200 domain (black boxes) that can be divided into 3 subtypes (HIN A, HIN B and HIN C) based on their conserved amino acid content ${ }^{69}$. Both human and murine PYHIN genes are localized 
at chromosome 1q21-23. The human PYHIN family (A) consists of 4 members and of these only IFI16 contains two HIN200 domains (HIN A and HIN B) while MNDA and IFIX/Pyhin1 only contain one HIN A domain. AIM2 is phylogenetically distinct from the other family members with both its pyrin and HIN domain (HIN C) having the lowest sequence similarity to the pyrin and HIN domains of other PYHIN proteins. The murine family $(\mathbf{B})$ is much more diverse, predicted to consist of at least 13 members. As with human IFI16, IFI204 (p204) is the only murine PYHIN protein consisting of a pyrin and two HIN200 domains (HIN A and HIN B). Some conflicting data has been reported for BC094916. Thus it may be truncated (marked by $\uparrow$ ) to give rise to a final gene product lacking a HIN B domain. IFI202 lacks a pyrin domain, but has two HIN200 domains (HIN A and HIN B) and was shown to be inhibitory. As with human AIM2, murine AIM2 is phylogenetically distinct from the other family members. 
Table 1. Characteristics of cytosolic DNA sensors triggering type I IFN induction.

\begin{tabular}{|c|c|c|c|c|c|}
\hline $\begin{array}{l}\text { DNA } \\
\text { sensor }\end{array}$ & $\begin{array}{l}\text { Pathogens } \\
\text { sensed }\end{array}$ & Ligands tested & Cell type & $\begin{array}{l}\text { Signalling } \\
\text { molecules } \\
\text { involved }\end{array}$ & Ref \\
\hline DAI & $\begin{array}{l}\text { HCMV, HSV-1, } \\
\text { S. pneum. }\end{array}$ & $\begin{array}{l}\text { AT-rich B-DNA, } \\
\text { Viral, bacterial, } \\
\text { mammalian gDNA } \\
500 \mathrm{bp}>>100 \mathrm{bp}>75 \mathrm{bp}\end{array}$ & L929 & $\begin{array}{l}\text { TBK1, IRF3, } \\
\text { RIP1, RIP3, } \\
\text { NF-кB }\end{array}$ & 21,70 \\
\hline $\begin{array}{l}\text { RNA pol } \\
\text { III }\end{array}$ & $\begin{array}{l}\text { L. pneum.?, } \\
\text { Adenovirus, } \\
\text { EBV, HSV-1? }\end{array}$ & $\begin{array}{l}\text { AT-rich B- DNA, } \\
\text { EBERs }\end{array}$ & $\begin{array}{l}\text { HEK293, HeLa, } \\
\text { MEF, mBMDM, } \\
\text { RAW264.7, } \\
\text { human } \\
\text { monocytes, } \\
\text { L929, Mutu III }\end{array}$ & $\begin{array}{l}\text { RIG-I, } \\
\text { MAVS, } \\
\text { TBK1, DDX3, } \\
\text { IRF3 }\end{array}$ & 26,27 \\
\hline LRRFIP1 & $\begin{array}{l}\text { L. mono., } \\
\text { VSV }\end{array}$ & $\begin{array}{l}\text { dsDNA, dsRNA } \\
\text { AT-rich B-DNA, } \\
\text { GC-rich Z-DNA }\end{array}$ & $\begin{array}{l}\text { murine } 1^{\mathrm{o}} \mathrm{PM}^{\mathrm{a}}, \\
\text { RAW264.7 }\end{array}$ & $\begin{array}{l}\beta \text {-catenin, } \\
\text { p300, IRF3 }\end{array}$ & 32 \\
\hline DHX9 & HSV & CpG-A & human pDCs & $\begin{array}{l}\text { MyD88, } \\
\text { NF-кB (p50) }\end{array}$ & 33 \\
\hline DHX36 & HSV & CpG-B & human pDCs & $\begin{array}{l}\text { MyD88, } \\
\text { IRF7 }\end{array}$ & 33 \\
\hline $\begin{array}{l}\text { IFI16 } \\
\text { /p204 }\end{array}$ & HSV-1 & $\begin{array}{l}\text { dsDNA } \\
\text { Sequence-indep. } \\
70 \mathrm{bp}>>50 \mathrm{bp}\end{array}$ & $\begin{array}{l}\text { THP1, } \\
\text { RAW264.7, } \\
\text { MEF, HeLa, } \\
\text { BMDM }\end{array}$ & $\begin{array}{l}\text { STING, } \\
\text { TBK1, IRF3, } \\
\text { NF-кB }\end{array}$ & 29 \\
\hline Ku70 & $\mathrm{HSV}-2 \mathrm{G}$ & $\begin{array}{l}\text { dsDNA, ssDNA, } \\
\text { sequence-indep. } \\
\text { 500bp }>>50 \mathrm{bp}\end{array}$ & $\begin{array}{l}\text { HEK293, human } \\
\text { MDM }^{\text {b }} \text { murine } \\
\text { spleen cells }\end{array}$ & $\begin{array}{l}\text { IRF1, IRF7 } \\
\text { IFN } \lambda 1\end{array}$ & 34 \\
\hline
\end{tabular}

${ }^{a} \mathrm{PM}$, peritoneal macrophages; ${ }^{\mathrm{b}} \mathrm{MDM}$, monocyte-derived macrophages 
Table 2. Comparison of innate sensing of DNA by ALRs compared to RNA by RLRs.

\begin{tabular}{|l|l|l|}
\hline & ALRs & RLRs \\
\hline Ligand & Self or non-self dsDNA & Viral RNA \\
\hline IFN induction & IFI16, p204 & RIG-I, Mda5 \\
\hline Inflammasome activation & AIM2, IFI16 & RIG-I \\
\hline Negative regulation & p202 & LGP2 \\
\hline Viral evasion & M13L, pUL83 & NS1, K7 \\
\hline Pathogen sensed & Virus and bacteria & Virus and bacteria \\
\hline Cellular compartment & Cytosol (and nucleus?) & Cytosol \\
\hline
\end{tabular}

